\title{
Radial migration in barred galaxies
}

\author{
P. Di Matteo ${ }^{1}$, M. Haywood ${ }^{1}$, F. Combes ${ }^{2}$, B. Semelin ${ }^{2}$, \\ C. Babusiaux ${ }^{1}$ and A. Gomez \\ ${ }^{1}$ GEPI, Observatoire de Paris, CNRS, Université Paris Diderot, \\ 5 place Jules Janssen, 92190 Meudon, France email: paola.dimatteo@obspm.fr \\ ${ }^{2}$ LERMA, CNRS, UPMC, Observatoire de Paris, \\ 61 Avenue de l'Observatoire, 75014 Paris, France
}

\begin{abstract}
In this talk, I will present the result of high resolution numerical simulations of disk galaxies with various bulge/disk ratios evolving isolated, showing that:

- Most of migration takes place when the bar strength is high and decreases in the phases of low activity (in agreement with the results by Brunetti et el. 2011, Minchev et al. 2011).

- Most of the stars inside the corotation radius (CR) do not migrate in the outer regions, but stay confined in the inner disk, while stars outside CR can migrate either inwards or outwards, diffusing over the whole disk.

- Migration is accompanied by significative azimuthal variations in the metallicity distribution, of the order of 0.1 dex for an initial gradient of $\sim-0.07$ dex $/ \mathrm{kpc}$.

- Boxy bulges are an example of stellar structures whose properties (stellar content, vertical metallicity, $[\alpha / \mathrm{Fe}]$ and age gradients, ..) are affected by radial migration (see also Fig. 1).
\end{abstract}

Keywords. Galaxies: abundances, Galaxies: evolution, Galaxies: dynamics
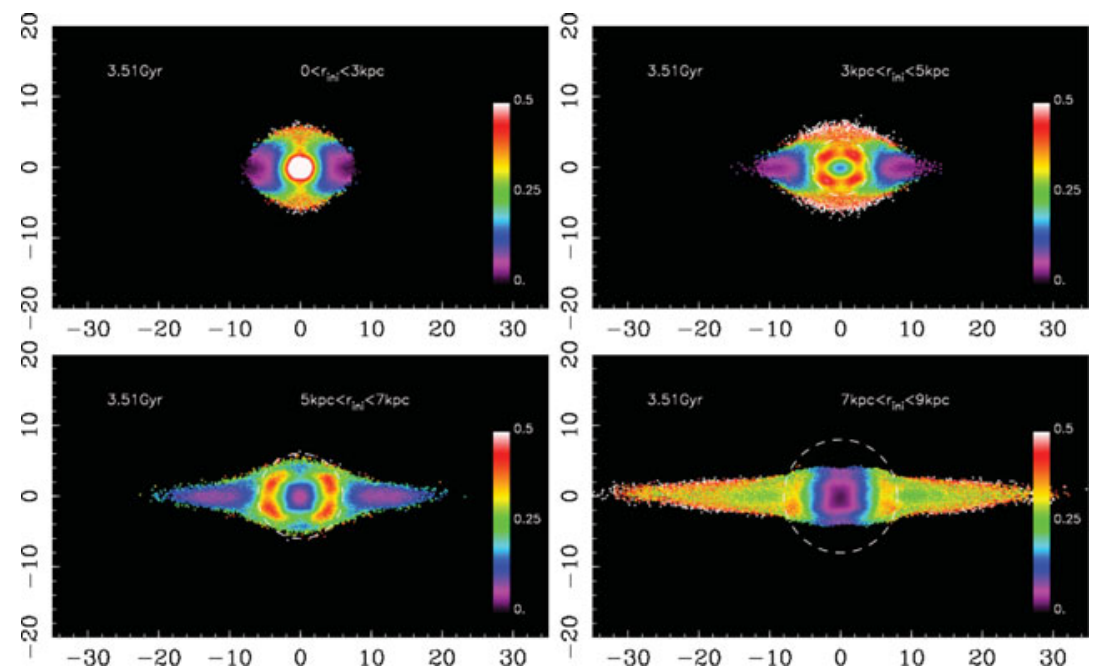

Figure 1. Fractional contribution of stars born at different radii (top-left panel: $r_{i n i}<3 \mathrm{kpc}$; top-right panel: $3 \mathrm{kpc}<r_{i n i}<5 \mathrm{kpc}$; bottom-left: $5 \mathrm{kpc}<r_{i n i}<7 \mathrm{kpc}$; bottom-right: $7 \mathrm{kpc}$ $\left.<r_{i n i}<9 \mathrm{kpc}\right)$ to the stellar content of a boxy bulge and disk. Axis units are in kpc.

\section{References}

Brunetti, M., Chiappini, C., \& Pfenniger, D. 2011, A $\mathscr{G} A$ 534, 75

Minchev, I., et al. 2011, A\&\&A 527, 147 